\title{
Current status and challenges of using geometric tolerance information in intelligent manufacturing systems
}

\author{
Hirpa G. Lemu
}

Received: 12 January 2014/ Accepted: 24 January 2014/Published online: 2 March 2014

(c) Shanghai University and Springer-Verlag Berlin Heidelberg 2014

\begin{abstract}
Recent development in computer-based manufacturing and inspection has necessitated extended knowledge and usage of geometric tolerances as carriers of design intent. The aim of applying geometrical tolerances in design is to provide function-oriented precise description of part geometry where the conventional size tolerance system fails to address. In view of the current development of computer-aided systems, applying geometric tolerances opens a new research front. This article examines the challenges in applying geometric tolerance information to carry the design intent to other downstream manufacturing processes and intelligently integrate the whole system. Based on the observed practical capabilities and literature studies, it is concluded that the current computer-aided design (CAD) systems cannot effectively provide the appropriate use of geometric tolerances. This article highlights the existing challenges and proposes a scheme of algorithm development for appropriate use of tolerance symbols and conditions at the design specification stage. This, in the long run, enables the CAD model to carry the design intent and opens a window of opportunity for intelligently integrating manufacturing systems.
\end{abstract}

Keywords Geometric tolerance · ISO $1101 \cdot$ ASME $\mathrm{Y} 14.5 \mathrm{M} \cdot$ Intelligent manufacturing $\cdot$ Coordinate measuring machine $\cdot$ Computer-aided tolerating

H. G. Lemu ( $\varangle)$

Department of Mechanical and Structural Engineering and Materials Science, University of Stavanger, Stavanger, Norway e-mail: Hirpa.g.lemu@uis.no

\section{Introduction}

Mechanical design is by nature a synthesis work intended to give precise description of the part on engineering drawings using pictures, texts, numbers and symbols. The design specifications transfer the design intent stated as the geometrical and material characteristics, the critical function relationships among features in part and the assembly requirements. The design specifications are not unique in nature, but valid within permissible ranges that should not come in conflict with the functional requirements. In order to guarantee the intended functions and assembly relationships, tolerances are specified, which define the permissible variations from the ideal part given in the drawing. This is because it is almost impossible to produce a part with perfect size and form as indicated in the drawing. Size variations are given by the size tolerances that are traditionally accepted to be sufficient to guarantee fulfilment of functional requirements. Taguchi [1] came up with another view indicating that any deviation from the ideal geometry was a loss of functionality. Thus, by specifying dimensions and size tolerances on drawings, designers convey the design intent concerning the shape requirements for manufacturing and inspection. Similarly, geometric tolerances convey the design intent concerning form and functional requirements.

Nowadays manufacturing industries are under intense pressure from the ever-increasing competition demanding products to be produced within tighter tolerances, shorter time to market and more accurate communication with design intent [2]. This has made many companies to recognize the importance of having competence in tolerating their drawings with geometric tolerances. The existing manufacturing practice on the workshop floor indicates that there are two main challenges to make smooth flow of 
design intent. Primarily, the practical use of the rules, symbols and concepts of geometric tolerances is not well established. In view of the extended use of computers in almost every activity involved in a product's life cycle, the ability to represent a design model that is complete, consistent, meaningful and unambiguous is absolutely necessary. As the interpretation of the standards is not easy, companies are doing significant investment on training their engineers and technicians so that the geometric tolerance concepts given by the standards are well understood and applied. Secondly, computer representation of geometric tolerances, i.e., the symbols and textual rules, demands development of complex algorithms. It is crucial that the design representations including the tolerance information allow easy modification and design optimization. The ability to transfer both the dimensional model data and the associated tolerances including the providing functional requirements from computer-aided design (CAD) model is important for future progress of computeraided inspection systems. In the process of developing common neutral files for data transfer, recent development in the standard for the exchange of product model data is widely expected as the most promising tool to solve the constraints on transfer of design data.

The objective of this article is to highlight the research and application challenges of using geometric tolerance information as a carrier of design intent to intelligently integrate the activities in the manufacturing process. On the one hand, the article addresses the concern in the possible misunderstanding of the relatively complicated international standards of geometric tolerances when implemented in the industry. On the other hand, as a result of the dynamic situation due to regular updates of the standards and the wider room for different interpretations of the geometric tolerance parameters, it is clearly observed that the established codes of measurement practice of geometric tolerances are insufficient. Variation in inspection techniques obviously leads to different results. Thus parts to be rejected may be accepted or parts to be accepted can not pass the quality control. The lack of unified and consistent measurement practice is more of concern when softwarebased inspection tools is used and mainly driven by commercial interests that dominate their availability and the frequency of software and hardware updates.

\section{Literature review}

Intelligent integration of design intent to manufacturing, assembly and inspection implies that the tolerance information is computer readable. This has not been an easy task as geometric tolerance information is expressed by a symbolic language using various symbols and texts. Driven by the industrial need and the computerization progresses, current research focuses on modeling and representation of the tolerance information to make the geometric tolerances readable by computer. For instance, Zhao et al. [3] reported that research in modeling and representation of geometric tolerances could be classified as system dependent and system independent. This classification is based on whether the tolerance modeling takes place within or out of the geometric modeling environment. Accordingly, the representation technique used will depend on the geometric modeling and representation. The solid models can be based on the implicit (unevaluated) form such as constructive solid geometry (CSG) representation or boundary representation (B-rep). While the CSG expresses the solid object symbolically using some basic primitives and Boolean operations, the B-rep uses topology and structure of vertices, edges and faces to represent a solid surface. The algorithms for these techniques are mainly developed to express the mathematical representation of the solid models, and they are less applicable to represent the functional requirements that geometric tolerances stand for. However, some researchers have attempted to incorporate geometric tolerance information into the geometry representations using CSG [4], B-rep solid model representation [5] and hybrid B-rep/CSG representation [6]. B-rep models represent the object explicitly and thus offer good visualization of the geometry. On the other hand, CSG models contain information about the model in unevaluated form, thus good visualization requires evaluated forms into explicit vertices, edges and surfaces. Hybrid B-rep/CSG representation is favoured in modern CAD systems in order to benefit from both representations.

Among the mathematical algorithms proposed to establish the mathematical basis representing tolerances, we find computational geometry based approach [7, 8], variational geometry method $[6,9]$ and graph-based representation [5, 10]. Many geometric tolerance parameters including the additional specification conditions on the drawings, graph-based and variational geometry representations obviously result in complicated algorithms. Graphbased algorithms for complex geometries and product assemblies with many parts give rather unconceivable network of graphs. Roy and Li [11] also studied the challenges of incorporating geometric tolerances in the progress of CAD process in order to automate the transfer of design intent and indicated that a complete tolerance system should

(i) be compatible with current solid modeling system;

(ii) represent standard tolerance practices;

(iii) support automated tolerance analysis and synthesis.

This study highlights that the variational representation is more appropriate with respect to current solid modeling 


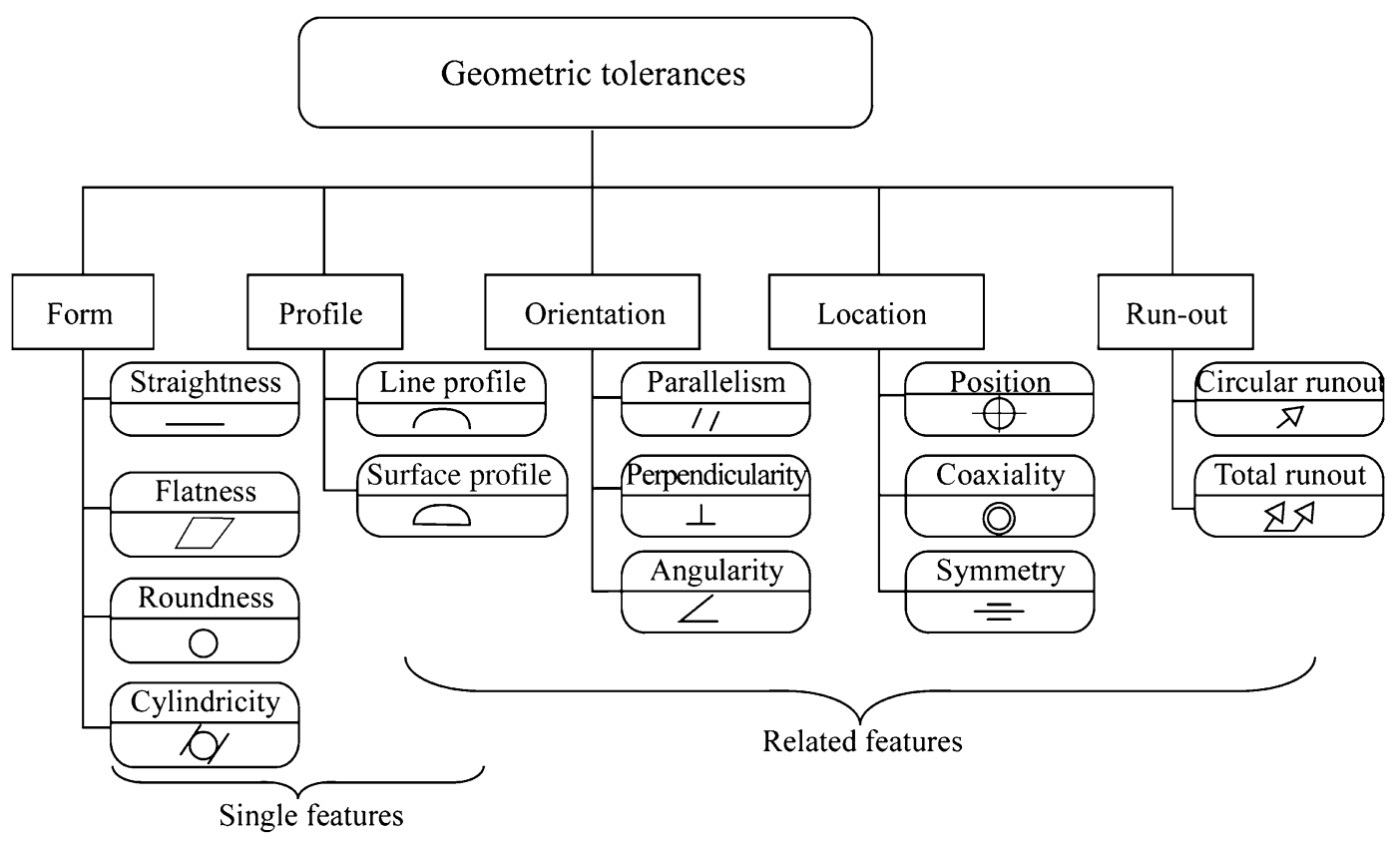

Fig. 1 Overview of the basic geometric tolerance parameters and symbols

systems. It allows variation of the boundary surface of the surface model within specified tolerance zone. On the other hand, as discussed later in this article, the existing CAD systems have no capability to make automated tolerance analysis at least at the level of advising the designer on correct use of the tolerance symbols and application conditions.

\section{Overview of geometric tolerancing principles}

According to ISO 1101 [12] and ASME 14.5 [13], geometric tolerance is defined as "...an international language of symbols placed on technical drawings to adequately describe the allowable variation of part geometry." In other words, the purpose of geometric tolerances is to establish smooth communication between the users of the standard. Accordingly, the geometric tolerance language uses welldefined set of symbols, rules, definitions and conventions to enable the required smooth communication.

The geometric tolerance characteristics and symbols are basically categorized into three main groups: form, orientation and location. However, this paper would like to group the parameters (14 in number) into five categories. This is becoming the practice in most recent publications and textbooks in the field. Figure 1 shows the geometric tolerance categories, the parameters under each category and the symbols. The geometric characteristic symbol $(S)$, i.e., one of the symbols in Fig. 1, and the tolerance value $(t)$ are given in a rectangular tolerance frame with at least two compartments (for single features) and up to five compartments (for related features). A typical tolerance frame with examples of modifying parameters is illustrated in Fig. 2.

Two major principles, the maximum material condition (MMC) principle and the independence principle (ISO 8015), lay the foundation of modern tolerating principle using geometric tolerances. While the MMC principle attempts to contain the form variations within the worst case boundary, the independence principle provides clear distinction between size and form tolerances unless a specific relationship is defined.

\section{Standardization of geometric tolerances}

The guidelines of specifying geometric tolerances are standardized in two main international standards: ASME and ISO standards. ASME adopts the American Y14.5 national standard (previous ANSI standard) and introduces some other concepts, definitions, rules and symbols. The most recent main revision of the standard is ASME Y15.5: 2009 [13]. Geometric tolerances in ISO are given by ISO 1101 in which the recent updated version is ISO 1101:2004. In addition, ISO has issued a series of documents on geometric tolerances and other engineering drawing standards. According to this standard [12], a geometric tolerance applied to a feature defines the tolerance zone within which the feature shall be contained. ASME 14.5 is based on the MMC principle while ISO standards adopt both the MMC and independence 


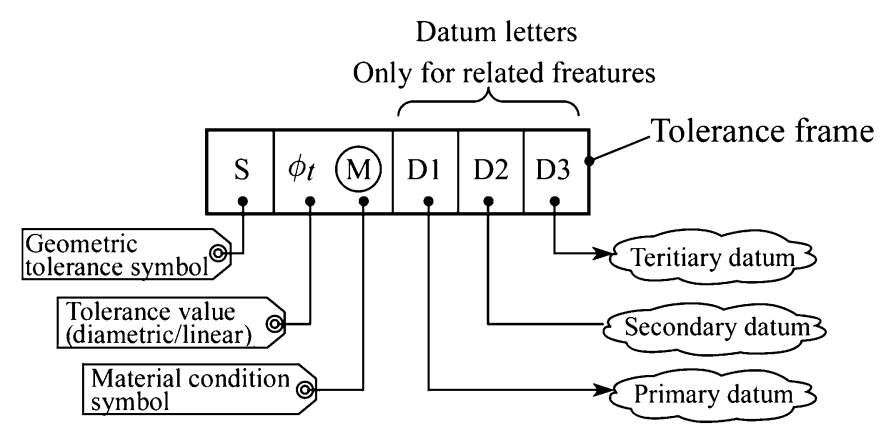

(a)

\begin{tabular}{|c|l|}
\hline Symbol & Description \\
\hline (Ait) & Datum target \\
\hline (M) & Maximum material requirement \\
\hline (D) & Least material requirement \\
\hline (P) & Projected tolerance zone \\
\hline (E) & Envelope requirement \\
\hline (T) & Tangent plane \\
\hline$\phi$ & Diameter \\
\hline$s \phi$ & Sphere diameter \\
\hline
\end{tabular}

(b)

Fig. 2 Illustration of reference frame a and examples of modifying symbols b

Table 1 Terminology difference between ASME 14.5M: 2009 and ISO 1101: 2004

\begin{tabular}{ll}
\hline ASME $14.5 \mathrm{M}$ & ISO 1101 \\
\hline Basic dimension & Theoretical exact dimension (TED) \\
Feature control frames & Tolerance frame \\
Variation & Deviation \\
True position (TP) & Theoretical exact position \\
Reference dimension & Auxiliary dimension \\
\hline
\end{tabular}

Table 2 Symbols specified in ASME 14.5M: 2009 (not in ISO 1101: 2004)

\begin{tabular}{|c|c|c|}
\hline Symbol & Designation & Interpretation \\
\hline. & All round & $\begin{array}{l}\text { Tolerance applicable all round to the } \\
\text { bounded line shown by the tolerance } \\
\text { specification }\end{array}$ \\
\hline $\begin{array}{l}\text { ALL } \\
\text { OVER }\end{array}$ & All over & Applicable everywhere (to all surfaces) \\
\hline AVG & Average & $\begin{array}{l}\text { Arithmetic mean (specially for flexible } \\
\text { parts) }\end{array}$ \\
\hline CR & $\begin{array}{l}\text { Controlled } \\
\text { radius }\end{array}$ & Radii at all points within tolerance \\
\hline$\longleftrightarrow$ & Between & Tolerance applicable to a limited segment \\
\hline (T) & Tangent & $\begin{array}{l}\text { Applicable to the tangent (contacting) } \\
\text { element }\end{array}$ \\
\hline ST & $\begin{array}{l}\text { Statistical } \\
\text { tolerating }\end{array}$ & Statistical tolerance control (STC) required \\
\hline
\end{tabular}

principles. In terms of the used symbols and concepts in both standards, there are about $90 \%$ similarity between them [14]. The differences involve both terminology and symbology, where the ASME 14.5M specifies additional symbols that do not exist (not yet defined) in ISO 1101 . Some selected examples are given in Tables 1 and 2.

Due to the difficulties in mathematical modeling and representation, implementing the standards in the seamless linking of $\mathrm{CAD}$ and manufacturing (CAD/CAM) system has not been straightforward. Furthermore, the standards are frequently revised with introduction of some new concepts, symbols and conventions while some are removed from the standards. For instance, Fig. 3 shows some of the design conventions that used to be part of former practices as given by ISO 1101:1983, but omitted in the 2004 version. The measures clearly improve the existing ambiguities in the standard. Closer review of the literature also shows that the research on how geometric tolerance information can be represented is not progressing with the same speed as the representation and integration of geometric modeling and manufacturing data.

\section{Tolerance zone}

The tolerance zone specifies the region within which the part feature (axis, point, line, surface or median plane) deviation is constrained. This zone has different forms partly depending on the type of the geometric tolerance parameter and partly basing on the specifications given in the tolerance frame. In other words, some of the geometric tolerance properties are defined by specific form of the tolerance zone while some are dictated by the specifications given by the designer. For instance, a flatness tolerance is defined only between two parallel lines (in 2D) or three parallel planes (in 3D). Thus modification of the tolerance zone in the tolerance frame is not allowed. In a similar way, a cylindricity error is bounded by a condition that points of a revolution surface are equidistant from a common axis, thus its tolerance zone is bounded by two concentric cylinders. The tolerance feature may be of any form or orientation within the defined tolerance zones unless restricted by other specifications [15]. Figure 4 shows the various forms of the available tolerance zones for geometric tolerances, both in 2D and 3D. Most tolerance zones are in $3 \mathrm{D}$, but the $2 \mathrm{D}$ versions that are the projections of the 3D space on a plane are more intuitionistic in tolerance analysis. 

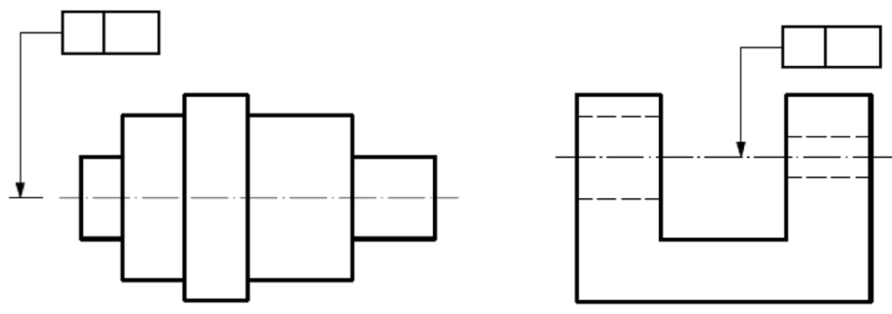

(a)

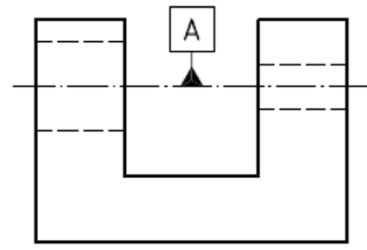

(b)

Fig. 3 Examples omitted former practices of indicating a geometric tolerances and $\mathbf{b}$ datum triangle with datum letter on common axes
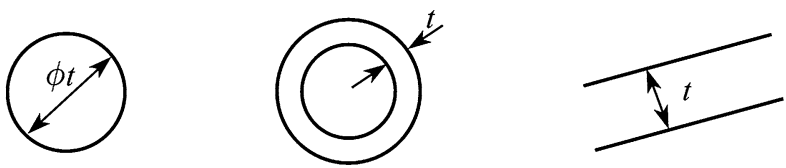

(a) 2D tolerance zones (circle, concentric circles and parallell lines)
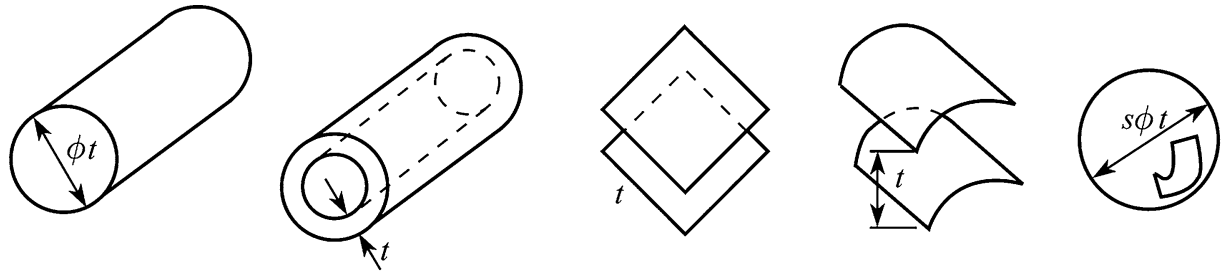

(b) 3D tolerance zones (cylindrical region, coaxial cylinders, parallell planes/surfaces and spherical region)

Fig. 4 Sample forms of tolerance zones for geometric tolerances

Tolerance zone formulation is one of the early attempts to achieve computer-based representation of geometric tolerances. Requicha [16] indicated the challenges of incorporating tolerance information, which are essential for design analysis, process planning, assembly planning and other applications into modern solid modeling systems.

In this work, the tolerance zone is used as a means of defining a minimum region of space so that the feature is said to be produced according to the design intent when all points of the tolerance feature are constrained within the limits of the tolerance zone. The diversity of parameters and application conditions cause difficulties to define a universal representation technique. This has forced researchers to focus on a particular geometric tolerance or a few of them with certain common characteristics. For instance, Teck et al. [17] developed a general method to define tolerance zones of form deviation using three parameters corresponding to the three degrees of freedom of a planar surface (one translation and two rotations). The method is not only limited to form deviations but also applied only to planar/flat surfaces. Others [18-21] focused on representation of circularity error.

According to the technical note of Moroni and Petró [22], the minimum zone criterion can essentially be formulated as a non-linear optimization that mostly leads to local minima or even non-convergence. Using this criterion, many optimal theories are proposed [23] including combinatorial optimization approaches.

\section{Geometric tolerances and assembly requirements}

As stated earlier, geometric tolerance information is given in a drawing primarily to fulfill functional requirement. Additional purposes include assembly requirement (e.g., interchangeability of products), manufacturing requirement and inspection requirement are conveyed by geometric tolerance specification.

Functional requirement cannot be seen isolated from other requirements, particularly assembly. This is because the assembly condition influences the product's functionality. A tight tolerance may be good for some parts but the assembly process can be difficult. Relaxed tolerance can also influence both function and ease of assembly. This is particularly true when the tolerance of a component exceeds its permissible designed value that leads to either difficulty of assembly work or ease of 
assembly but poor performance. Cost is directly related with either side of the conditions.

Assembly process of a part is affected by dimension and geometric tolerances. Location and orientation tolerances in particular are carriers of design intent on how a feature on the part is located and oriented both in the manufacturing process and the assembly. Dimensions of features with location and orientation tolerances are indicated in the drawing using data that are given partly with respect to assembly requirements and partly to indicate how the feature is constrained in space. Considering that producing the exact feature is impossible and in order to ease the assembly requirement, geometric tolerances are often given with material conditions that fall into three categories: MMC designated by (M), least material condition (LMC) designated by $(\mathbb{C})$, and regardless of material condition that serves as a default value when the other two material conditions are not specified.

\section{Coordinate measuring machines for inspection of geometric tolerances}

The progress in computer-based product modeling, numerical control (NC) and the precision in the machining technologies nowadays has made inspection using hard gauges unsuitably. As a result, the use of a coordinate measuring machine $(\mathrm{CMM})$ is becoming a natural choice of the future. The role of CMM as a tool of product inspection in a manufacturing system is increasing as a result of the growing importance of qualifying products to given specified dimension and form. However, implementing the tool in an efficient and systematic way to smoothly communicate design intent to inspection and product control is not straightforward. On top of the sophistication of the geometric tolerance as a language of engineering drawing, the technology of CMM to measure and interpret the data is demanding. This includes, among others,

(i) developing suitable and easy way to use measuring techniques and enabling the measured set of data to accurately represent the part to be inspected;

(ii) developing tolerance verification algorithms that are consistent with the existing standards of geometric tolerances like ISO 1101 and ASME 14.5M.

The advantage of CMM as a tool of product inspection is not only the fact that it improves inspection quality, but also enables control of complex geometries such as free form surfaces and reduces the inspection time. The last mentioned is attributed to the elimination of complex jigs and fixtures' setup that are common problems in the traditional inspection method.
Though CMM is a precise machine being used as a standard inspection tool now, the application is hampered by problems related to issues like sampling scheme, probing strategies and identifying systematic errors and random noises in the measured data [24]. The sampling and probing work is mostly done by experience that leads to variations in the inspection quality and reliability. Correct sampling techniques highly influence the reference substitute geometry generated in order to estimate the error in the tolerance zone. To tackle the second problem, two types of algorithms are widely mentioned as suitable algorithms for CMM based inspections: (i) least-square type algorithms $[25,26]$ that compute the sum of squared errors; (ii) the minimum zone type algorithms [22]. The least-square type algorithm is widely used in commercial CMM-based inspections and claimed to be efficient, but inconsistent with the ASME 14.5M standard. Thus, inspections based on such algorithms can result in rejection of good parts or acceptance of poor parts. The minimum zone algorithm, on the other hand, is claimed to be better consistent with standards, but previous studies [27, 28] show that the algorithm is more computationally demanding specially for complex geometries.

\section{Indications for future research}

Geometric tolerances have been implemented in engineering drawings as carriers of design intent for over half a century. They can ease the communication between design to manufacturing and inspection. The geometric tolerances are symbolic languages involving many parameters and application conditions, and that this language is still under dynamic change in the standardization front, which makes it less understood in the industry—at least requires regular updating.

The symbolic representation and the regular changes being undertaken by standardization organizations have also created certain level of challenges on research to develop consistent algorithms that contribute to smooth transfer of design intent with less human intervention. As most of the geometric tolerance parameters have mutual interdependence, the algorithm development can be eased by studying the common characteristics.

From a designer's point of view, based on the author's own experience, the challenges are directly related with understanding the existing standards of geometric tolerance information specification of design that in general includes:

(i) identifying the tolerance type in regard to the functional and assembly requirements; 


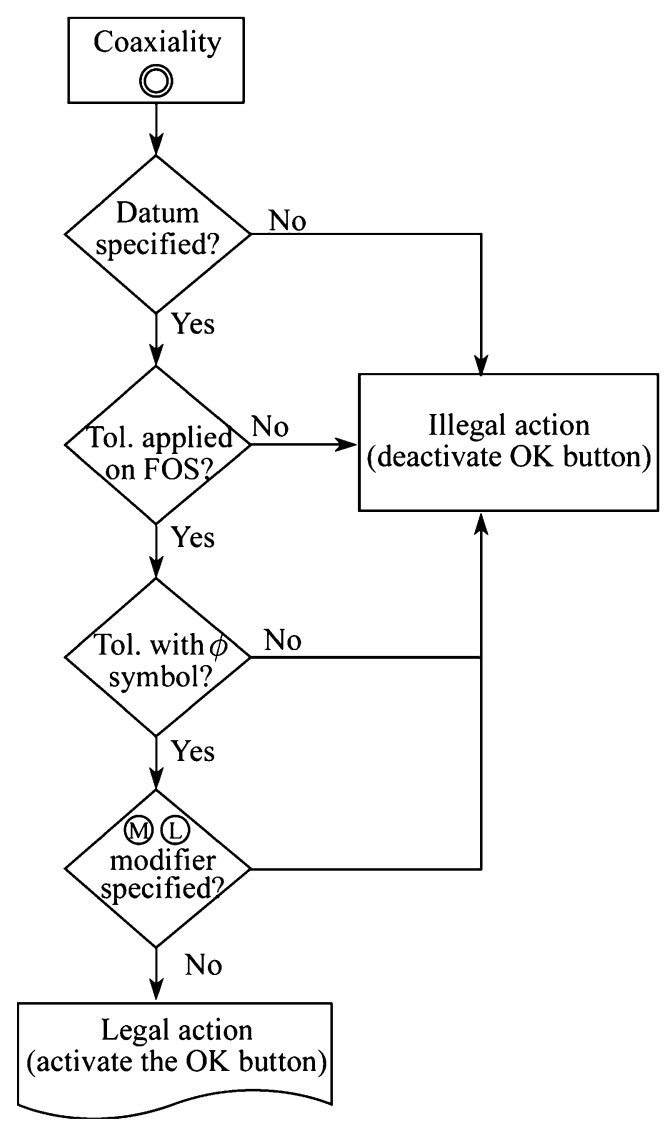

(a)

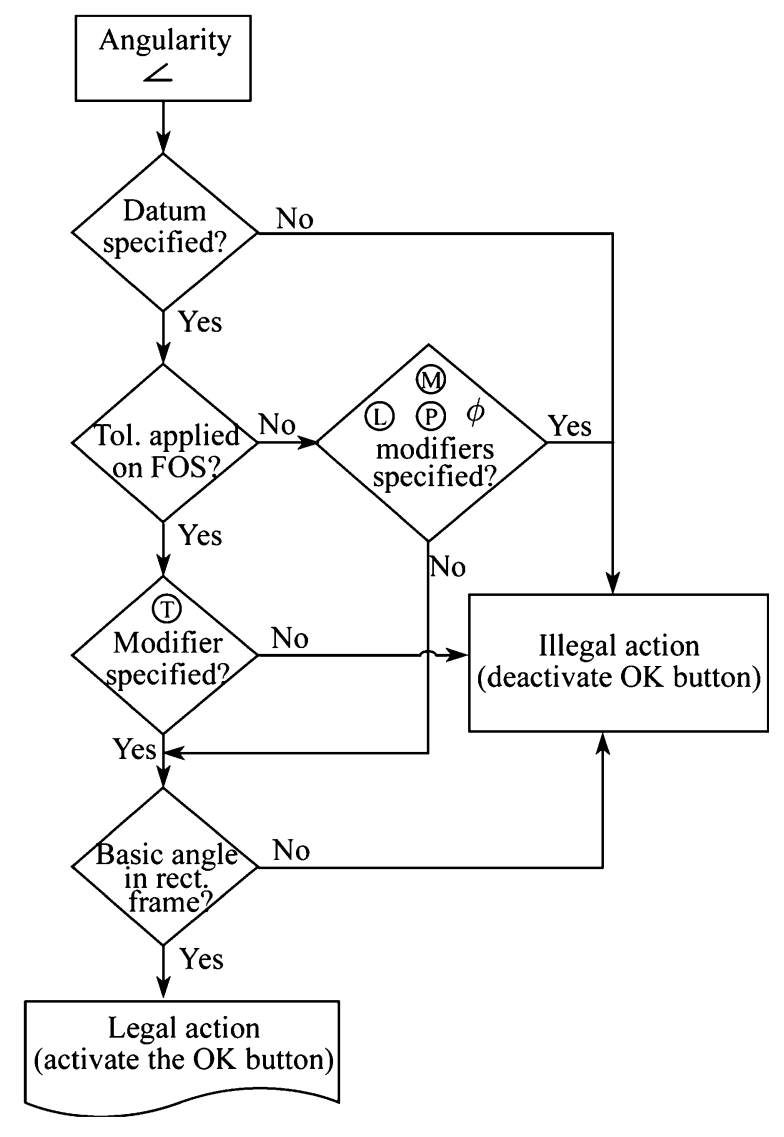

(b)

Fig. 5 Flowchart of a validity control for coaxiality $\mathbf{a}$ and angularity $\mathbf{b}$

(ii) identifying how the specifications are indicated in the drawing according to accepted/established language given by the standards;

(iii) specifying the legal conditions and determining the value of the tolerance that is achievable by the existing competence.

Makelainen and Heilala [29] claimed that existing commercial computer-aided tolerancing (CAT) tools offered tolerance analysis and synthesis capabilities either within independent software packages, or more commonly through integration with commercial CAD systems. However, the recent experience, over a decade after this claim, has shown that the existing CAT assists the designer mainly with the symbols and some other tools to specify tolerances, while the main decisions are still mostly done manually. The correct selection of the design codes and the effective interpretation of the measured results from CMM depend on the competence and knowledge of the user. To give precise definition of geometric tolerances and make them be the carriers of design intent, it is sought that there exists conceptual assistance in the form of warning/error messages, for instance when illegal symbols or tolerance specifications are committed. In other words, validity control at the design phase lacks the support. This paper proposes the development of validity control algorithms that can be incorporated with CAD tools and gives guidance of tolerance specification in 2D design drawings. Figure 5 shows sample flowcharts of the proposed tool for validity control. The benefit of such tools will not only ensure the application of appropriate conditions of geometric tolerances, but also make the learning curve of design engineers steep.

The systems operating intelligently are expected to perform with no or minor human intervention. The challenges of geometric tolerances to function in an intelligent system are observed even when CMM is used, where the output results of the machine are still interpreted by the inspector. As depicted by two output results shown in Fig. 6, the machine highly contributes with visualization tools such as color and mark indicating the tolerance state of the measured values and indication in the tolerance zone. However, this is still far from intelligent system. 


\begin{tabular}{|c|c|c|c|c|c|c|c|}
\hline Size & MM & & & $\not 13.25$ & $0.15 /-0.1$ & & \\
\hline MEAS & NOMINAL & $+\mathrm{TOL}$ & $-\mathrm{TOL}$ & DEV & OUTTOL & BONUS & \\
\hline 13.402 & 13.250 & 0.150 & -0.100 & 0.152 & 0.002 & 0.050 & \\
\hline 106.413 & 106.500 & 0.200 & 0.200 & -0.087 & 0.000 & 0.287 & \\
\hline Position & MM & & & \begin{tabular}{|l|l|}
$\phi$ & $\varnothing 0$ \\
\end{tabular} & \begin{tabular}{|l|l|l|}
1 (ii) & $\mathrm{A}$ & $\mathrm{B}(\mathrm{M})$ \\
\end{tabular} & & \\
\hline MEAS & NOMINAL & $+\mathrm{TOL}$ & $-\mathrm{TOL}$ & DEV & OUTTOL & & BONUS \\
\hline 10.025 & 0.000 & 0.100 & & 0.025 & 0.000 & & 0.050 \\
\hline 0.287 & 0.000 & 0.000 & & 0.287 & 0.000 & & 0.000 \\
\hline Size & MM & & & $\varnothing 16.35$ & $0.15 /-0.1$ & & \\
\hline MEAS & NOMINAL & $+T O L$ & -TOL & DEV & OUTTOL & BONUS & \\
\hline 16.377 & 16.350 & 0.150 & -0.100 & 0.027 & 0.073 & 0.000 & \\
\hline 13.402 & 13.250 & 0.150 & -0.100 & 0.152 & 0.002 & 0.050 & \\
\hline Position & MM & & & \begin{tabular}{|l|l}
$\oplus 10$ \\
\end{tabular} & \begin{tabular}{|l|l|}
0.03 (D) & $H \otimes 1$ \\
\end{tabular} & & \\
\hline MEAS & NOMINAL & $+\mathrm{TOL}$ & $-\mathrm{TOL}$ & DEV & OUTTOL & & BONUS \\
\hline 0.000 & 0.000 & 0.030 & & 0.000 & 0.000 & & 0.000 \\
\hline 0.050 & 0.000 & 0.000 & & 0.050 & 0.000 & & 0.000 \\
\hline
\end{tabular}

Fig. 6 Sample outputs of a CMM tool indicating state values and tolerance zones

\section{Conclusions}

This paper assessed current status and application challenges of geometric tolerance information in mechanical drawing of parts from the view point of intelligently transferring design intent to manufacturing and inspection. The article focuses on highlighting both the application challenges of the existing international standards (ISO/ ASME) on the workshop floor and the research challenges to develop efficient, unambiguous and consistent algorithms that facilitate the transfer of design intent to the downstream processes with no or minor human interference.

The computer-aided tools in the future of design engineering are expected to establish a system that operates intelligently and performs better than before. However, the existing machines and operations in the area are still unable to mimic some basic human capabilities such as adjusting appropriately to the dynamic environment, understanding some of the human readable symbols and texts, etc. It is only when such human actions and other natural reactions are "learned" that the system is said to be intelligent. This requires both hardware and software used in the area that have the ability to adapt to the dynamic changes. Investigating the extent of the use of geometric tolerance information to realize the required manufacturing system intelligence and the accompanying challenges is the main goal of the study reported in this article.

The challenges are observed both on the upstream and downstream side. On the upstream side, i.e., at the design specification phase, the design tools fall short of giving conceptual support to the designer when inappropriate geometric tolerance symbols and conditions are used. On the downstream side, CMM tools are providing formidable support to the inspection work by allowing inspection of complex geometries and reducing the inspection time. However, interpretation of the output results still depends, to a certain extent, on the competence of the inspector. Future research should particularly focus on the upstream side of the manufacturing system to further develop algorithms and find how the developed results can be incorporated into future CAD systems.

\section{References}

1. Taguchi G (1986) Introduction to quality engineering: designing quality into products and processes. Asian Productivity Organization, Tokyo 
2. Cogorno GR (2006) Geometric dimensioning and tolerancing for mechanical design. McGraw-Hill Professional, New York

3. Zhao X, Pasupathy TM, Wilhelm RG (2006) Modelling and representation of geometric tolerances information in integrated measurement processes. Comput Ind 57:319-330

4. Requicha AAG, Chan SC (1986) Representation of geometric features tolerances and attributes in solid models based on constructive geometry. IEEE J Robot Autom RA-2(3):156-166

5. Guilfor J, Turner JD (1993) Representational primitives for geometric tolerancing. Comput Aided Des 25(9):577-586

6. Turner JU (1990) Relative positioning of parts in assembly using mathematical programming. Comput Aided Des 22(7):394-400

7. Samuel GL, Shunmugam MS (2000) Evaluation of circularity from coordinate and form data using computational geometric techniques. Precis Eng 24(3):251-263

8. Samuel GL, Shunmugam MS (1999) Evaluation of straightness and flatness error using computational geometric techniques. Comput Aided Des 31(13):829-843

9. Gupta S, Turner JU (1991) Variational solid modelling for tolerance analysis. In: Proceedings of ASME International Conference on Computer Engineering, CA, USA, pp 487-494

10. Tsai J-C, Cutkosky MR (1997) Representation and reasoning of geometric tolerances in design. Artif Intell Eng Des Anal Manuf 11:325-341.

11. Roy U, Li B (1999) Representation and interpretation of polyhedral objects II. Comput Aided Des 31:273-285

12. ISO 1101 (2004) Geometrical product specification (GPS)-tolerance of form, orientation, location and runout, 2nd edn. International Organization for Standardization, Geneva

13. ASME (2009) Dimensioning and tolerancing. ASME Standard Y14(5M):2009

14. Krulikowski A (1998) Fundamentals of geometric dimensioning and tolerancing, 2nd edn. Division of Thomas Learning Inc., Delmar

15. Green $P$ (2005) The geometrical tolerancing desk reference: creating and interpreting ISO standard technical drawings. Elsevier Ltd, Oxford
16. Requicha AAG (1983) Toward a theory of geometric tolerancing. Int J Robot Res 2(4):45-60.

17. Teck TB, Kumar AS, Subramanian V (2001) A CAD integrated analysis of flatness in a form tolerance zone. Comput Aided Des 33:853-865

18. Zhu LM, Ding H, Xiong YL (2003) A steepest descent algorithm for circularity evaluation. Comput Aided Des 35(3):255-265

19. Dhanish PB (2002) A simple algorithm for evaluation of minimum zone circularity error from coordinate data. Int J Mach Tool Manu 42(14):1589-1594

20. Wen X, Xia Q, Zhao Y (2007) An effective genetic algorithm for circularity error unified evaluation. Int J Mach Tool Manu 46: $1770-1777$

21. Venkaiah N, Shunmugam MS (2007) Evaluation of form data using computational geometric techniques-part I: circularity error. Int J Mach Tool Manu 47:1229-1236

22. Moroni G, Petró S (2008) Geometric tolerance evaluation: a discussion on minimum zone fitting algorithms. Precis Eng 32: 232-237

23. Anthony GT, Anthony HM et al (1996) Reference software for finding Chebyshev best-fit geometric elements. Precis Eng 19(1):28-36

24. Gou JB (1999) A geometric theory of form, profile and orientation tolerances. Precis Eng 23:79-93

25. Forbes AB (1989) Least-square best fit geometric elements. Technical report of National Physical Laboratory, Middlesex, UK

26. Gou JB, Chu YX, Li ZX (1998) On the symmetry localization problem. IEEE Trans Robot Autom 14(4):540-553

27. Wang Y (1992) Minimum zone evaluation of form tolerances. ASME Manuf Rev 5(3):213-220

28. Kanada T, Suzuki S (1993) Application of several computing techniques for minimum zone straightness. Precis Eng 15(4): 274-280

29. Makelainen E, Heilala J (2001) Assembly process level tolerance analysis for electromechanical products. In: Proceedings of the IEEE international symposium on assembly and task planning, 2001, pp 405-410 